\title{
Antipsychotika verschlechtern die Stress-Symptome bei Palliativpatienten
}

\author{
Hydratation, Reorientierung und soziale Unterstützung durch \\ Familienangehörige sind die wichtigsten Supportivmaßnahmen \\ bei Delirium. Können Antipsychotika Distress-Symptome zusätz- \\ lich abschwächen?
}

\) Disstress und Verhaltensauffälligkeiten im Rahmen eines Deliriums werden häufig mit Antipsychotika behandelt. Kontrollierte Studien zeigten eine Verbesserung der deliranten Symptomatik, allerdings auf methodisch nicht sehr hohem Niveau. Leitlinien empfehlen Antipsychotika lediglich für die Behandlung von starkem Distress und Verhaltensstörungen, die auf andere Maßnahmen nicht ansprechen. Eine große placebokontrollierte Studie sollte nun die stressreduzierenden Effekte von Risperidon und Haloperidol bei Delirpatienten in der Palliativsituation untersuchen.

In der prospektiven randomisierten Studie wurden 247 Palliativpatienten untersucht, die doppelblind, altersadjustiert und dosistitriert entweder ora- les Risperidon, orales Haloperidol oder Plazebo erhielten. Die Medikation wurde über 72 Stunden gegeben, bei Bedarf war Midazolam erlaubt. Zusätzlich wurden die Patienten mit Allgemeinmaßnahmen wie Hydratation sowie Seh-, Hör-und Reorientierungshilfen unterstützt. Primäres Studienziel war die durchschnittliche Gruppendifferenz des Delirium-Symptomscores, berechnet als Summe der Verhaltens-, Kommunikations- und Wahrnehmungsitems aus der „Nursing Delirium Screening Scale“ zu Behandlungsbeginn und -ende.

Das Ergebnis ist überraschend: Statt die Symptome zu bessern, verschlechterten beide Neuroleptika den Distress im Vergleich zu Plazebo: Im Risperidon-Arm stieg der Delirium-Score um durchschnittlich $0,48 \quad$ Einheiten (95\%-Konfidenzintervall [KI]: 0,090,86; $p=0,02$ ), im Haloperidol-Arm um durchschnittlich 0,24 Einheiten (95\%KI: 0,06-0,42; $p=0,009)$. Der Midazolam-Verbrauch war in allen drei Gruppen vergleichbar. Extrapyramidale Nebenwirkungen traten bei den Neuroleptika-behandelten Patienten erwartungsgemäß häufier auf als unter Plazebo.

Bei Palliativpatienten mit deliriumbedingtem psychischen Distress, so das Fazit, verschlechtern Risperidon und Haloperidol im Vergleich zu Plazebo die Symptome und erhöhen die Rate extrapyramidaler Störungen. Die Patienten sollten daher primär eine Supportivtherapie erhalten.

(bkr)

\section{Quelle}

Agar MR. E.cacy of oral risperidone, haloperi-dol, or placebo for symptoms of delirium among patients in palliative care. A randomized clinical trial. JAMA Intern Med 2017;177:34-42

\section{Cybercoping: Bloggen bis zum letzten Atemzug}

\section{In Ruhe und Zurückgezogenheit sterben war gestern. Heute schreiben sich viele das Leid in aller Öffentlichkeit von der Seele. Sie kommen dadurch mit der Krankheit oft besser zurecht.}

" Hallo. Ich heiße Dmitrij Panov und ich werde bald sterben. Klingt komisch, ist aber so." Mit diesen Worten beginnt der 25-jährige Marburger seinen Blog „Sterben mit Swag“. Die letzten neun Monate seines Lebens breitet er öffentlich seine Gedanken und Gefühle aus.

Bloggen ist populär geworden, auch das Bloggen über Krankheit und Tod. Social Media sind längst in die Krankenzimmer und Hospize vorgedrungen. Das Web 2.0 als interaktive Bühne vor anonymem Publikum, das die Selbstentblößung erleichtert, das mal applaudiert, mal aufmuntert, mal kritisiert, mal pöbelt. In einer Welt, in der viele bereit sind, alle Details ihres Le- bens auf Facebook vorzuführen, mag die Hemmschwelle für ein öffentliches Krankheitstagebuch gering sein, der Krebsblog ist dann häufig nur die Fortführung der "Timeline“ im öffentlichen Raum. So war es etwa bei der 2016 gestorbenen Moderatorin Miriam Pielhau: Sie machte einfach ihre Facebookseite öffentlich. Ihr letzter Eintrag wurde über 6.000-mal „geliked“.

Doch einen öffentlichen Krankheitsblog starten auch Patienten, die sich für Social Media zuvor nur wenig interessiert haben. Was also bewegt Menschen, plötzlich vor großem Publikum über ihre Körperfunktionen und -dysfunktionen zu plaudern?

\section{Ähnlich wie expressives Schreiben}

Neben dem Verlangen, ein Vermächtnis zu hinterlassen, kann das Bloggen ein Weg sein, mit der Krankheit besser zurechtzukommen. Kommunikationswissenschaftler um Stephen Rains und David Keating aus Tucson/USA sehen eine gewisse Ähnlichkeit dieses "Cybercopings" mit expressivem Schreiben, das bei der Bewältigung von Lebenskrisen helfen soll. In einer Metaanalyse von 150 Studien aus dem Jahr 2006 zeigten sich positive Auswirkungen auf Psyche und Gesundheit bei Personen, die sich das erlebte Leid von der Seele schrieben (Psychological Bulletin 2006; 132, 823). Das expressive Schreiben löst nach dem Inhibitionsmodell unterdrückte Gedanken und Emotionen, die sich negativ auf die Gesundheit auswirken, und fördert die 